\title{
Non-intrusive Appliance Load Monitoring (NIALM) System with the Possibility for Users to Follow the Consumption of Individual Electricity Appliances from the Calendar
}

\author{
Mikko Tuomisto \\ VTT Technical Research Centre of Finland, Espoo, Finland \\ Email address: \\ mikko.tuomisto@vtt.fi
}

\section{To cite this article:}

Mikko Tuomisto. Non-intrusive Appliance Load Monitoring (NIALM) System with the Possibility for Users to Follow the Consumption of Individual Electricity Appliances from the Calendar. International Journal of Energy and Power Engineering. Vol. 5, No. 3, 2016 , pp. 129-132. doi: 10.11648/j.ijepe.20160503.16

Received: February 29, 2016; Accepted: March 8, 2016; Published: June 12, 2016

\begin{abstract}
This paper presents a technical system, which uses the non-intrusive appliance load monitoring (NIALM) method. The solution enables the end user to observe the electricity consumption of the individual appliances. By dividing electricity consumption to individual appliances, it improves the users' understanding of the overall energy use of the building. This way the owner of the building is able to intervene better with the energy consumption of the building. Observation of the electricity appliances is performed in a web application in a user interface calendar view.
\end{abstract}

Keywords: NIALM, Usability, Electricity Saving

\section{Introduction}

In the United States, buildings are responsible for $40 \%$ of the primary energy consumption and $73 \%$ of the electricity consumption [1]. Previous studies have shown that it is possible to reduce energy consumption of buildings by $10-15 \%$ by using better energy management [2].

The building owners are often unaware of how appliances and the behaviour of the occupants of the building affect the energy performance of the building [3]. Therefore it is positive that the number of electrical appliances, which automatically measure building energy consumption, is increasing. This way the owner of the building is able to intervene better with the energy consumption used by the building.

A good energy metering application increases the possibility to reduce the energy consumption of the building, but a poorly made application would be useless or misleading. For example, blindly trusting the GPS navigation has resulted in numerous car accidents [4]. The same also applies to the measurement of energy efficiency. It is important that the occupants of the building get as good overall picture as possible as to where the energy is consumed. Therefore by dividing electricity consumption information to individual appliances, it improves the users' understanding of the overall energy use of the building. Non-intrusive appliance load monitoring (NIALM) method enables to share this needed information to the occupants.

\section{Non-intrusive Appliance Load Monitoring (NIALM)}

\subsection{The Basics of NIALM}

One way to observe the electricity consumption of the users is a non-intrusive appliance load monitor (NIALM) method. NIALM method is based on the changes in the electricity grid caused by the electrical appliances. Non-intrusive means that the identification of the electricity consumption of the electrical appliances is centrally detected and not detected on an individual appliance analyser [5]. NIALM was invented originally by George W. Hart, Ed Kern and Fred Schweppe in the early 1980s [6]. VTT Reasearch Centre of Finland have done research on the NIALM subject since 1990, led by Hannu Pihala [7].

This paper will focus in utilising the Non-Intrusive Appliance Load Monitoring (NIALM) method for creating an appliance specific breakdown of the electricity 
consumption of the users. It is valuable for the users to receive information on how much each of their electrical appliance consume. The method is able to detect the changes in the electricity consumption load in each electrical appliance. Each electrical appliance, when turned on or turned off, creates an electrical load change to the electricity grid. The load change is used to express the electricity consumption of the individual electrical appliances. These spikes caused by the electrical appliances are called the fingerprints.

\subsection{Categorization of Electricity Consumption Profiles}

Electricity consumption profiles can be divided into four categories. The First category is the on/off category, which means that the source can only be switched on or off. The Second category includes electrical appliances that may have more positions. A good example of the adjustable electrical appliance is the stove. The Third category is formed by electrical appliances which can be turned on or off, but during the operation of the electrical appliance, they cause different consumption events. An example of an electrical appliance in the Third category is a washing machine, which follows the program during the operation. The Fourth category is formed by electrical appliances that are always on and do not cause any changes to electricity grid, and therefore cannot be identified. Figure 1 demonstrates the example electricity profile, generated by the load of the electrical appliance changes. [8]

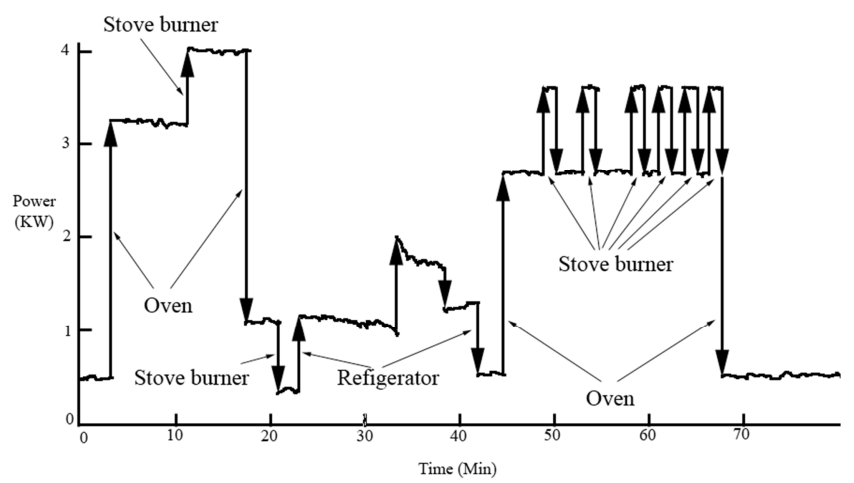

Figure 1. Electricity consumption profile. X-axis shows Time in minutes and Y-axis shows Power in $\mathrm{kW}[1]$.

\subsection{Identification of Single Electrical Appliances}

The identification of electrical appliances starts by teaching the system the electrical appliances, then the system can identify the electrical appliances automatically [8]. The system stores the starting time of the electrical appliance, the shutdown time of the electrical appliance, as well as the power used by the electrical appliance and then calculates the power consumption. By this way the electricity consumption profile and the using time of the electrical appliance can be formed. The fingerprints, formed by different electrical appliances, are expressed in figure 2 . The fingerprints forms clusters and are called detection areas.

\section{Description of the Tool}

In this project we used the EQLServer software, developed by MX Electrix, in which the different electrical appliances are identified based on their fingerprints illustrated in figure 3. Every cluster of appliances is linked to the specific appliance in the electricity grid. After all the electrical appliances of the electricity grid are linked, the application starts automatically identifying them, if an appliance is detected into the identification area. For practical reasons, the detection area is usually rectangle-shaped. In theory, every electrical appliance of the electricity grid is possible to be identified. At the moment the software is not fully automatic and works as a standalone solution. The software operates in the server, so the identification of the electrical appliances is done in the server. The system is planned to be developed so that the identification of the electrical appliances can be done via a web user interface. This will enable users to identify the electrical appliances of their own electricity grid by themselves by using the web interface. NIALM method algorithms used by the software are based on the study of Hannu Pihala [7].

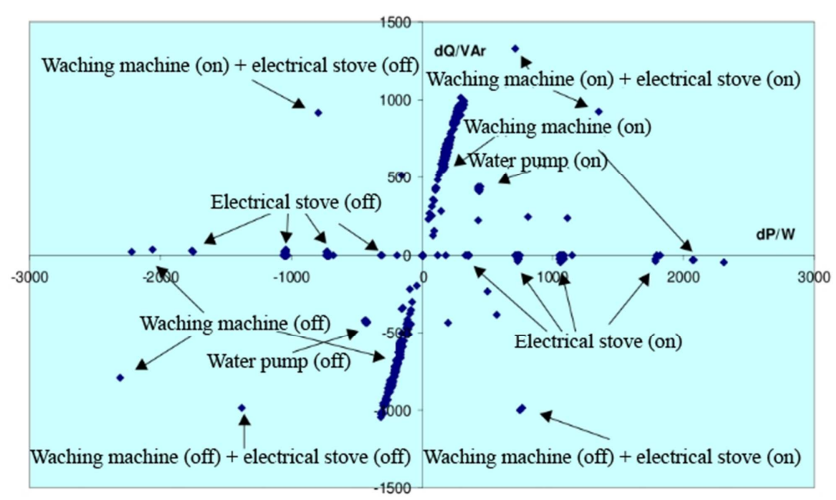

Figure 2. The Fingerprints of the electrical appliances [9].

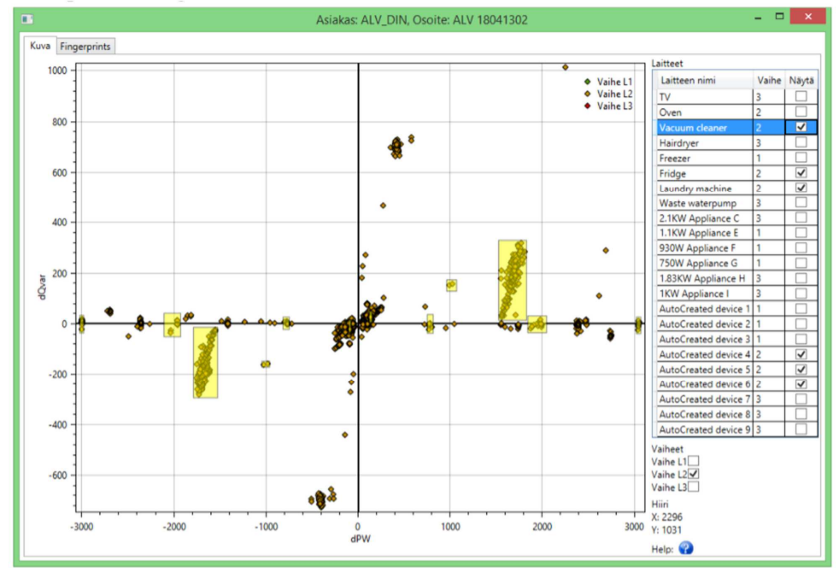

Figure 3. The fingerprints of the electrical appliances in MX Electrix EQLServer application [10].

\section{Demonstration of the Tool}

The purpose of this chapter is to describe the demonstration performed in Aalto University. The test 
facility space is located in the Second floor of a building in Otaniemi at the University of Aalto, which includes office facilities, as well as a coffee room with kitchen. The MX Electrix electricity meter is installed in the premises, which is used to detect changes in the electricity grid. The normal electricity meters are designed to measure only electricity consumption, but the installed meters are designed to measure the quality of the electricity. The electrical meter can be read and can be commanded remotely.

The NIALM method installed has certain limitations. The method is not able to detect electrical appliances with very small electricity consumption. Another limitation is that the electrical appliances, which are continuously on cannot be detected, as they do not cause an identified change. The Third constraint is that the system is not able to detect the electrical appliances that turn on or turn off slowly.

The identification and analysis of the electrical appliances take place in the server, by using EQLServer software, developed by MX Electrix. The server software automatically retrieves the information from the meter over the LAN network, because this is possible with the MX Electrix meters. The data is written to the data store into the VTT Technical Research Centre server, where data is permanently stored. The system is illustrated in figure 4.

Technical Research

Centre of Finland

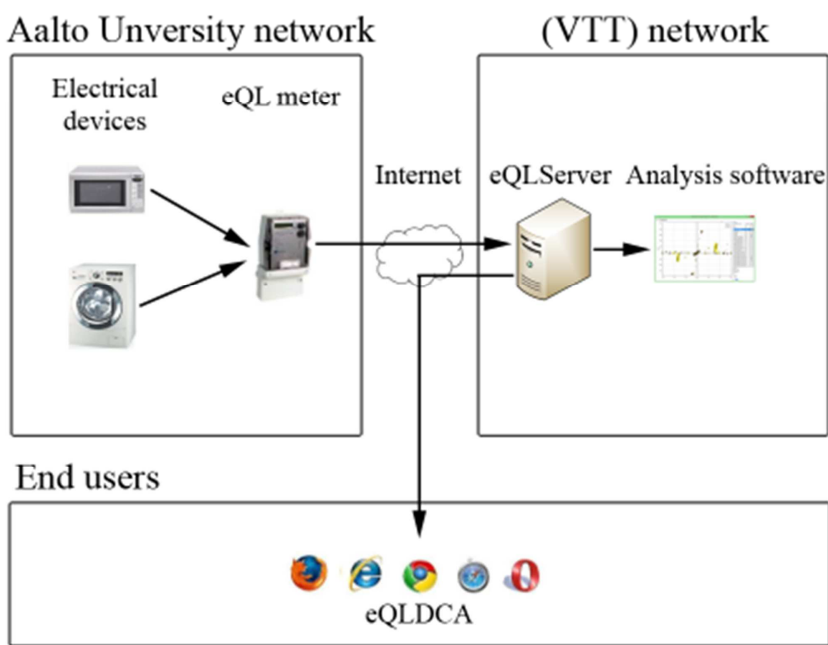

Figure 4. The technical framework of the system [10].

In addition, there is a web software that runs on a web browser, so the users have an easy access interface to the system from any computer. Figure 6 illustrates the Web interface where the electrical power consumption of the different electrical appliances is tracked. After the identification of the electrical appliances the eQLDCA software automatically marks the use of the different electrical appliances to the calendar, which is illustrated in figure 5 .

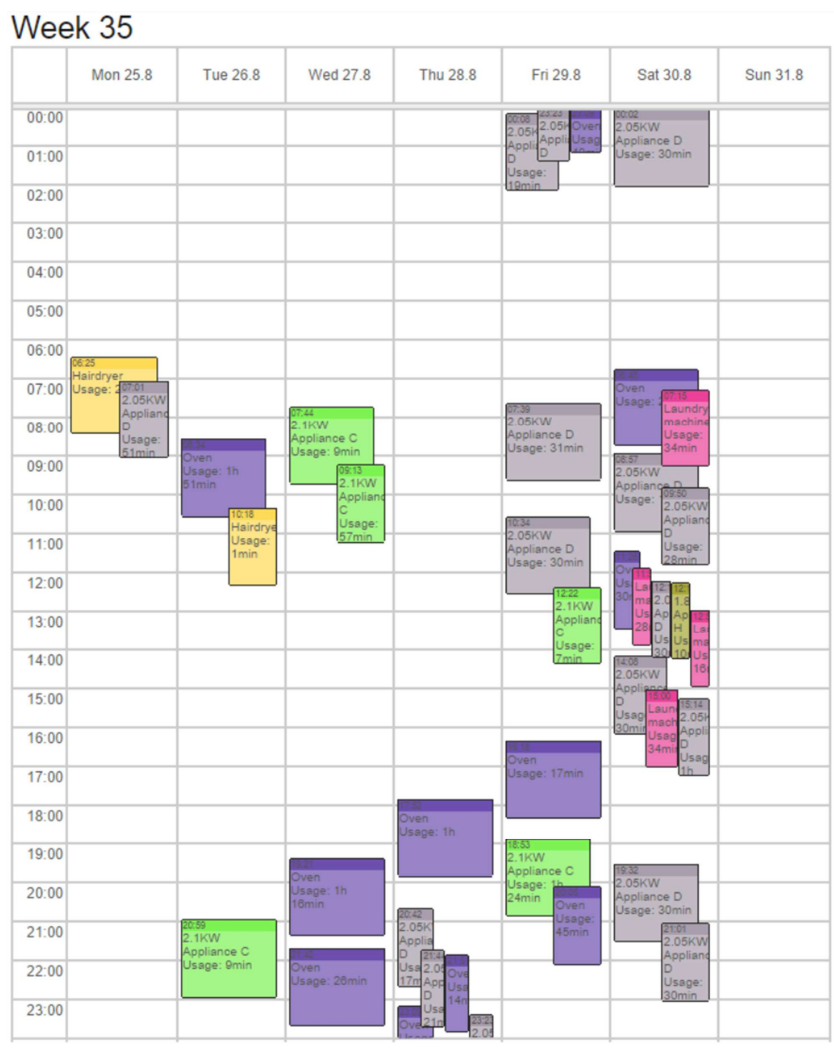

Figure 5. The one week use of the electrical appliances in the eQLDCA software [10].

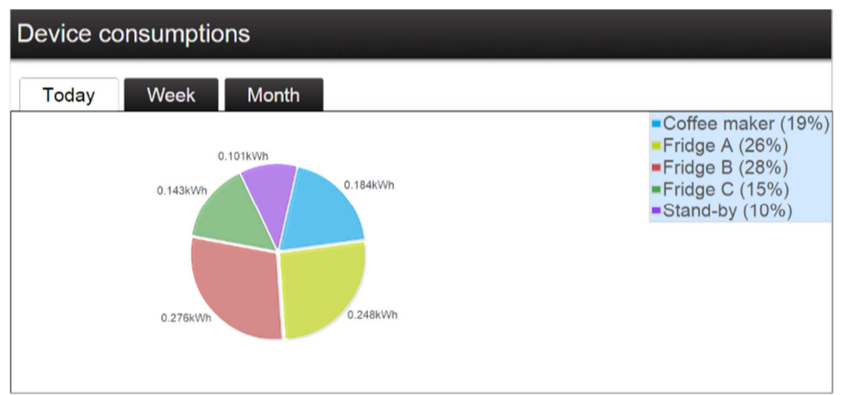

Figure 6. The user interface of the EQLServer software [10].

The system consists of the following technical components. The server is a virtual Windows Server where MySQL software is used as a databank. In addition, the server has MX Electrix EQLServer software, which has been made by using $\mathrm{C} \#$ and. NET programming languages. The web services use Apache and Django. The meter reading software is written with $\mathrm{C}++$ and Python programming languages.

\section{Future Plans}

The majority of the NIALM systems need a very complicated installation. When the technological NIALM system is installed in the electricity grid, an expert is needed. In addition to the physical installation of the NIALM system, the expert detects the devices which are used in the electricity grid. After this the expert links the changes in the electricity grid to the appliances which are used in the household. After installation, the technological NIALM system is able to 
automatically detect the appliances. The disadvantage is the fact that, if the new devices are brought to the network after the installation of the system, the detection of the new appliances is not updated to a technological NIALM system. The aim of the project is to create a system in which the user would be able to link electricity appliances to the changes, which the appliances create in to the electricity grid. The technological NIALM system would be adaptable. The experts would not be needed if appliances change in the electricity grid, because the user could make changes easily by using web interface.

\section{Conclusion}

This paper presented the technical system, which uses the non-intrusive appliance load monitoring (NIALM) method. The solution enables the end user to observe the electricity consumption of the individual appliances. By dividing the electricity consumption to individual appliances, it improves the users' understanding of the overall energy consumption of the building. This way the occupants of the building are able to intervene better with the energy consumption of the building. The studies have shown that it is possible to reduce energy consumption of the buildings by $10-15 \%$ by using better energy management. The impact in reducing electricity consumption by dividing electricity consumption information to individual appliances should be studied further.

\section{Acknowledgements}

I am grateful to Ph.D. Sami Karjalainen for commenting on this paper.

\section{References}

[1] M. Zeifman, K. Rothh, "Nonintrusive Appliance Load Monitoring: Review and Outlook," IEEE Transactions on Consumer Electronics, vol. 57, No. 1, February 2011

[2] K. Ehrhart-Martinez, K. A. Donnely, and J. A. Laitner, "Advanced Metering Initiatives and Residential Feedback Programs: a Meta-Review for Household Electricity-Saving Opportunities," Report E105, ACEEE 2010

[3] M. Marceau, R. Zmeureanu, "Nonintrusive load disaggregation computer program to estimate the energy consumption of major end uses in residential buildings," Elsevier Science, 2000

[4] L. Liikanen, "Extreme-user approach and the design of energy feedback systems," Helsinki Institute for Information Technology HIIT, Helsinki Univeristy of Technology TKK, Helsinki, 2009

[5] L. Farinaccio, R. Zmeureanu, "Using a pattern recognition approach to disaggregate the total electricity consumption in a house into the major end-uses," Elsevier Science S. A., 1999

[6] G. Hart, "Residential energy Monitoring and Computerized Surveillance via Utility Power Flows," IEE Technology and Society Magazine, 1989

[7] H. Pihala, "Non-intrusive appliance load monitoring system based on a modern kWh-meter", VTT Energy, VTT Publications 356, 1998

[8] G. Hart, "Nonintrusive Appliance Load Monitoring," Proceedings of the IEEE, VOL. 80, NO. 12, December 1992

[9] L. Vehviläinen "Seniorin sähkölaitteiden käytön valvonta sähkömittarilla", Aalto University, Master's thesis, 2011

[10] T. Saarinen, "Sähkölaitteiden sähköisten sormenjälkien hallinta", Tampere University of Technology, Master's thesis, 2015 